\title{
Grundlagen der elektrischen Energieversorgung
}

Von Dr.-Ing. habil. Gerhard Herold

Professor an der Universität Erlangen-Nürnberg

Mit 220 Bildern 
Die Deutsche Bibliothek - CIP-Einheitsaufnahme

\section{Herold, Gerhard:}

Grundlagen der elektrischen Energieversorgung / von Gerhard Herold.

Stuttgart : Teubner, 1997

ISBN-13: 978-3-519-06187-8

e-ISBN-13: 978-3-322-87190-9

DOI: $10.1007 / 978-3-322-87190-9$

Das Werk einschließlich aller seiner Teile ist urheberrechtlich geschützt. Jeder Verwertung außerhalb der engen Grenzen des Urheberrechtsgesetzes ist ohne Zustimmung des Verlages unzulässig und strafbar. Das gilt besonders für Vervielfältigungen, Übersetzungen, Mikroverfilmungen und die Einspeicherung und Verarbeitung in elektronischen Systemen.

(c) B. G. Teubner Stuttgart 1997

Gesamtherstellung: Präzis-Druck GmbH, Karlsruhe

Einbandgestaltung: Peter Pfitz, Stuttgart 


\section{Vorwort}

Die Versorgung mit Energie gehört zu den elementaren Bedürfnissen aller Menschen. Das Lebensniveau in den hochentwickelten Ländern der Erde konnte nur auf einer stabilen und leistungsfähigen energetischen Grundlage entstehen. Ohne Energie ist keine industrielle Produktion möglich, Energie verleiht Mobilität, erleichtert das Leben und ist das Fundament seiner Annehmlichkeiten. Weltweit unterscheidet sich der Pro-KopfVerbrauch an Energie um zwei bis drei Größenordnungen mit den entsprechenden Auswirkungen auf die Lebensbedingungen. Die Verringerung dieser Unterschiede bei schonendem Umgang mit allen verfuggbaren Energiequellen ist nicht nur eine humanitäre, sondern auch eine existentielle Aufgabe. Daher wird die Auseinandersetzung mit Energiefragen auf lange Sicht von erstrangiger Bedeutung bleiben.

Die elektrische Energie nimmt seit dem Beginn ihrer großtechnischen Nutzung im Prozeß einer nachhaltigen energetischen Entwicklung auf der Erde wegen ihrer besonderen Eigenschaften und nicht zuletzt wegen ihrer hervorragenden Anpaßbarkeit an die Erfordernisse des Verbrauchs eine Schlüsselposition ein. Wir stehen am Beginn einer Entwicklung, in der in Kombination von moderner Leistungs- und Informationselektronik intelligente elektrische Betriebsmittel mit bisher unbekannten Gebrauchswerteigenschaften für eine rationelle Energieübertragung, -verteilung und -anwendung geschaffen werden, die zum weiteren Ausbau dieser Position beitragen wird. Elektrische Energieversorgung ist eine herausfordernde Aufgabe mit Zukunft.

Das vorliegende Buch entstand aus meiner Vorlesung "Grundlagen der elektrischen Energietechnik", die ich nun zum vierten Mal vor Studenten der Elektrotechnik des fünften Semesters an der Universität Erlangen-Nürnberg gehalten habe. Bei ihrer Konzeption ließ ich mich von der Ansicht leiten, daß man in der Ausbildung den aktuellen Entwicklungen in der elektrischen Energietechnik am besten durch Konzentration auf wichtige Grundlagen Rechnung tragen kann. Einerseits sollten es Grundlagen für weiterführende Vorlesungen sein. Andererseits lag es in meiner Absicht, Hörern, die später andere Spezialisierungsrichtungen einschlagen, "energietechnisches" Denken nahezubringen und so Verständnis und Offenheit für diese grundlegenden und zukunftsbestimmenden Fragen zu wecken.

Das erste Kapitel dient dem Überblick. Der grundsätzliche Aufbau von elektrischen Energieversorgungssystemen wird aus den Eigenschaften der elektrischen Energie abgeleitet. Nach einer Vorstellung typischer Netzstrukturen werden die wichtigsten elektrischen Betriebsmittel aufgeführt. Das zweite Kapitel faßt die für die elektrische Energietechnik wichtigen Grundlagen der Wechselstromtechnik zusammen. Es dient als Vorbereitung für die Auseinandersetzung mit symmetrischen Drehstromnetzwerken.

In der elektrischen Energieversorgung dominiert ungebrochen die Drehstromtechnik. Ihre Bedeutung hat sich durch die Entwicklungen auf dem Gebiet der modernen Leistungselektronik weiter erhöht. Das dritte Kapitel behandelt daher die in der elektrischen Energietechnik gebräuchlichen Koordinatentransformationen für Dreiphasensysteme in ihren wechselseitigen Zusammenhängen mit dem Ziel, Hilfsmittel für eine anschauliche 
Beschreibung von Betriebsvorgängen in Drehstromsystemen bereitzustellen. Das Fundament fur diese Betrachtungen ist die Transformation von Dreiphasensystemen in Raumzeiger und Nullgrößen.

Der Prozeß der elektrischen Energieversorgung wird sowohl im Hinblick auf quantitative Aspekte als auch auf seine Qualität durch Größen wie den Wirkungsgrad, die Wirk-, die Schein- und die Blindleistung sowie den Leistungsfaktor beschrieben. Unter dem Stichwort "Energiequalität" ist das Interesse an diesem Problemkreis vor allem im Zusammenhang mit dem zunehmenden Einsatz von leistungselektronischen Anlagen in Energieversorgungsnetzen sehr hoch. Die Beschränkung auf Leistungsgrößen bei kosinusförmigen Strömen und Spannungen ist daher nicht mehr zeitgemäß. Andererseits ist die wissenschaftliche Diskussion über verallgemeinerte, von den Zeitfunktionen der Ströme und Spannungen unabhängige, Leistungsgrößen noch nicht abgeschlossen. Dieser Situation angepaßt wird hier der Versuch unternommen, die Leistungstheorie möglichst allgemein aufzubauen und dabei doch mit wenigen Begriffen und Kenngrößen, die auf eine physikalisch interpretierbare Grundlage zurückgeführt werden können, auszukommen. Zwangsläufig kann so der wissenschaftliche Bearbeitungsstand nicht in allen seinen Aspekten widergespiegelt werden.

Das letzte Kapitel beschäftigt sich mit Fragen der wirtschaftlichen Energieversorgung angefangen von den Gestehungskosten für elektrische Energie über die Lastverteilung bis hin zur wirtschaftlichen Auslastung elektrischer Betriebsmittel. Nicht zuletzt soll hier die Erkenntnis vermittelt werden, daß elektrische Energieversorgung nur dann wirtschaftlich gestaltet werden kann, wenn das komplexe System über große Zeiträume betrachtet wird. Die Ansätze des Integrated-Ressource-Planning begründen ihre Wirkungen auf einer Erweiterung der Grenzen über das eigentliche elektrische System hinaus. Sie lassen sich von der Erkenntnis leiten, daß die Energieprobleme nur durch Erfassung immer komplexerer Zusammenhänge gelöst werden können.

Ein Buch wie das vorliegende ist in gewisser Weise immer ein Gemeinschaftswerk. So bin auch ich vielen Menschen für ihre Hilfe bei seiner Erarbeitung zu Dank verpflichtet. Mein besonderer Dank gilt Herrn Dipl.-Ing. Christian Weindl, der bei der Korrektur und Nachrechnung weder Zeit noch Mühen gescheut hat. Auch Frau Ingrid Willner, Frau Gertraud Stumpf, Frau Johanna Biegel und Herrn Tobias Knoll schulde ich großen Dank dafür, daß sie meine Computermanuskripte in eine dem Leser zumutbare Form gebracht haben. Schließlich danke ich dem Teubner-Verlag für die gute und fördernde Zusammenarbeit. 


\section{Inhaltsverzeichnis}

\section{AUFBAU VON ELEKTRISCHEN ENERGIEVERSORGUNGSSYSTEMEN}

1.1 Energie und menschliche Entwicklung ................................................... 1

1.1.1 Zum Begriff der Energie ......................................................................... 1

1.1.2 Wichtige Primärenergieträger ........................................................... 2

1.1.3 Kulturelle Entwicklung des Menschen und Energienutzung ........................... 5

1.1.4 Energieverbrauch in den entwickelten Industrieländern ............................. 8

1.1.5 Energieversorgung in den Entwicklungsländern ........................................ 9

1.2 Eigenschaften der elektrischen Energie............................................... 10

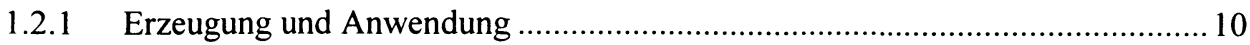

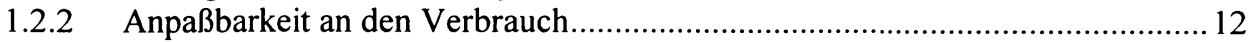

1.2.3 Unzureichende Speicherfähigkeit............................................................... 15

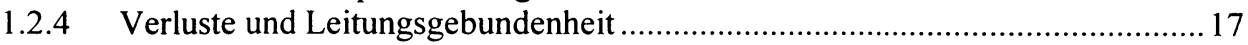

1.3 Elektrische Energieversorgungsnetze ....................................................... 20

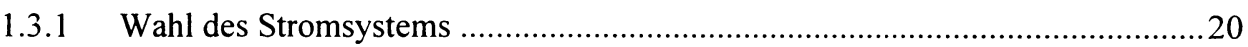

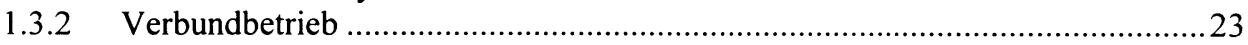

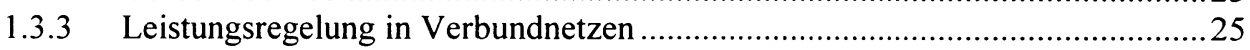

1.3.4 Struktur von elektrischen Energieversorgungsnetzen.................................28

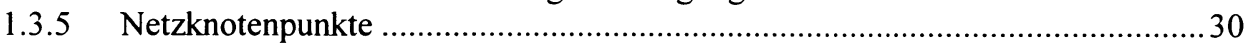

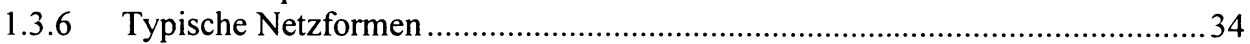

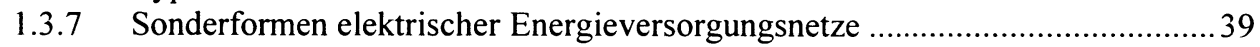

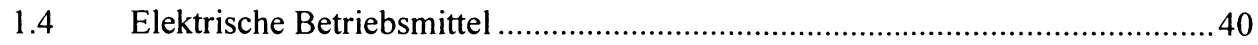

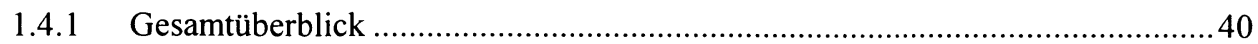

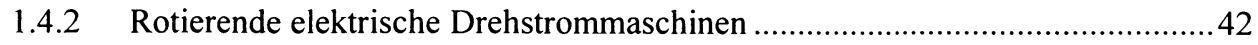

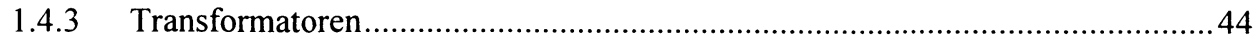

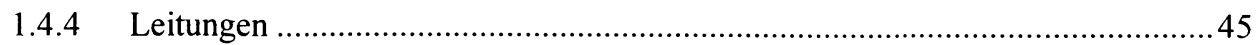

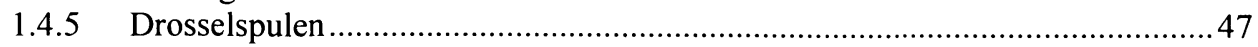

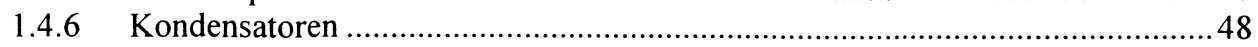

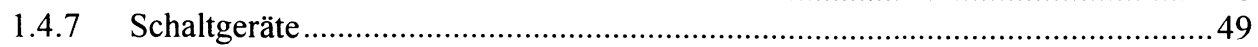

1.4.8 Sonstige Betriebsmittel und Elemente elektrischer Energieversorgungsnetze .......

\section{GRUNDLAGEN DER WECHSELSTROMTECHNIK}

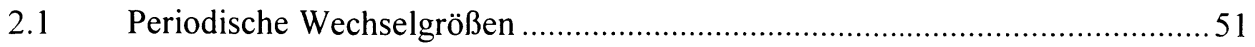

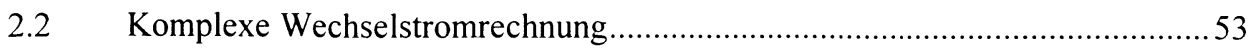


2.2.1 Komplexe Darstellung trigonometrischer Funktionen...................................53

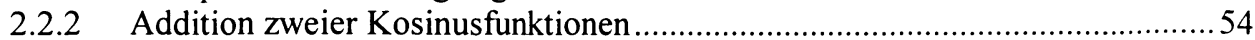

2.2.3 Darstellung kosinusförmiger Wechselgrößen durch Zeiger .............................55

2.2.4 Stationäre Ströme und Spannungen in Wechselstromkreisen...........................57

2.2.4.1 Kosinusförmiger Strom durch einen ohmschen Widerstand ...........................57

2.2.4.2 Kosinusförmige Spannung über einer idealen Induktivität ..............................58

2.2.4.3 Kosinusförmige Spannung über einer verlustbehafteten Induktivität................58

2.2.4.4 Kosinusförmiger Strom durch einen idealen Kondensator ...............................60

2.2.4.5 Kosinusförmiger Strom durch einen verlustbehafteten Kondensator ................60

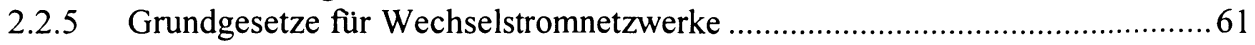

2.2.5.1 Kirchhoff'sche Sätze für kosinusförmige Wechselgrößen ..............................61

2.2.5.2 Ohmsches Gesetz für kosinusförmige Wechselströme ....................................62

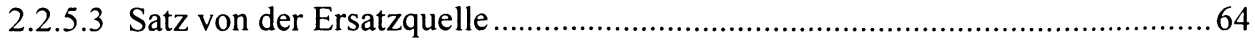

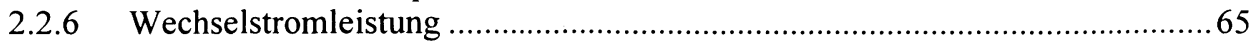

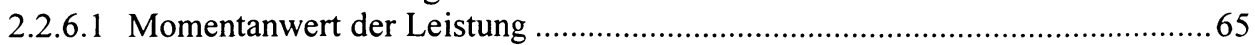

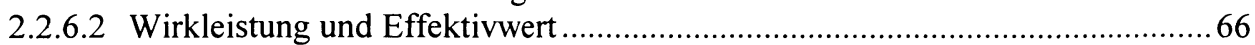

2.2.6.3 Leistung und Energie in einem verlustfreien Blindelement.............................67

2.2.6.4 Komplexe Darstellung der momentanen Leistung .......................................69

2.2.7 Kosinusförmige symmetrische Dreiphasensysteme.................................... 70

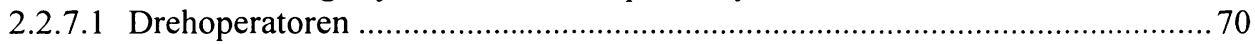

2.2.7.2 Sinusförmige symmetrische Ströme und Spannungen................................... 72

2.2.8 Vorzeichenfestlegungen und Zählpfeilsysteme ......................................... 76

2.3 Vierpole als Elemente von Wechselstromnetzwerken.................................... 80

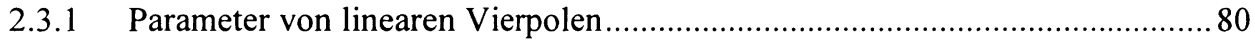

2.3.1.1 Leerlauf und Kurzschluß als spezielle Belastungsfälle des Vierpols ................80 80

2.3.1.2 Meßschaltungen zur Bestimmung der Vierpolparameter .............................. 82

2.3.1.3 Beschreibung des belasteten Vierpols ...................................................... 84

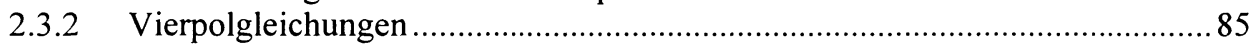

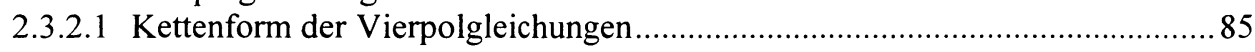

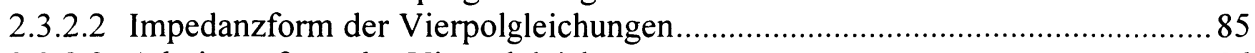

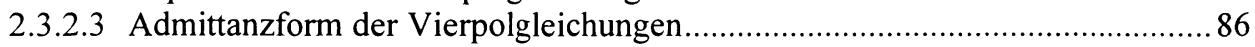

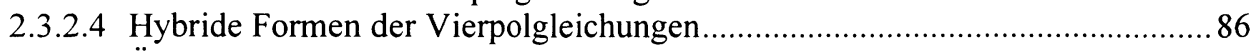

2.3.2.5 Änderung der Zählpfeilsysteme am Vierpol.................................................. 89

2.3.2.6 Leistungen an Vierpolen ........................................................................... 90

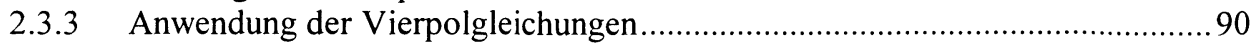

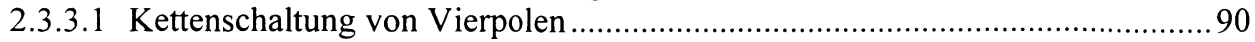

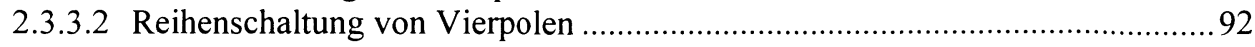

2.3.3.3 Parallelschaltung von Vierpolen ............................................................. 92

2.3.3.4 Reihen-Parallelschaltung und Parallel-Reihenschaltung von Vierpolen ............93

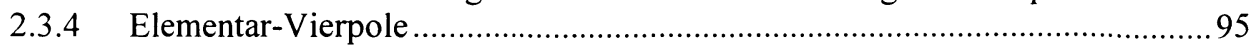

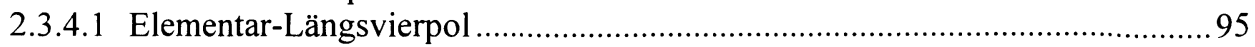

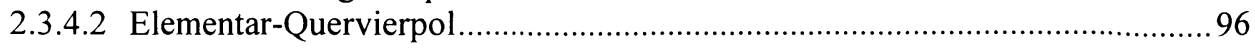

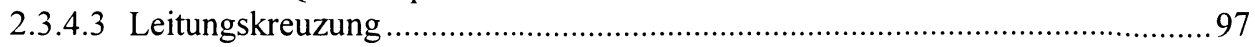

2.3.4.4 Synthese von Vierpolen aus Elementar-Vierpolen....................................... 97 
2.3.4.5 Grenzfälle der Belastung von Vierpolen in der elektrischen Energie-

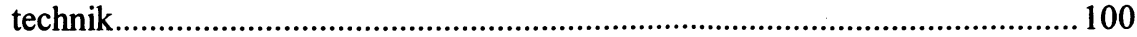

2.3.5 Homogene Vierpolketten ......................................................................... 101

2.3.5.1 Kettengleichung eines symmetrischen Vierpols............................................... 102

2.3.5.2 Kettengleichung einer Kette aus $\mathbf{n}$ symmetrischen Vierpolen ........................... 103

2.3.5.3 Spezielle Belastungsfäle der Vierpolkette...................................................... 104

2.3.6 Homogene Leitung................................................................................... 105

2.3.6.1 Ersatzschaltung eines Leitungselementes differentieller Länge ........................ 105

2.3.6.2 Leitungsgleichungen bei kosinusförmigen Strömen und Spannungen ............. 106

2.3.6.3 Wellenwiderstand, Übertragungsmaß und natürliche Leistung ........................ 108

2.3.6.4 Spezielle Betriebszustände der homogenen Leitung ....................................... 109

2.3.6.5 Ersatzschaltung und Zeigerdiagramm der kurzen Leitung ……….....................111

2.3.7 Vierpole mit induktiver Kopplung zwischen den Toren. ....................................113

2.3.7.1 Der mit einer Spule verkettete Fluß ……………....................................... 113

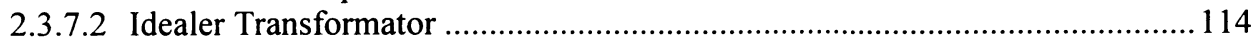

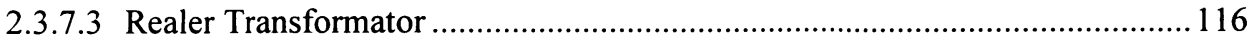

2.3.7.4 Messung der Parameter der Transformator-Ersatzschaltung............................119

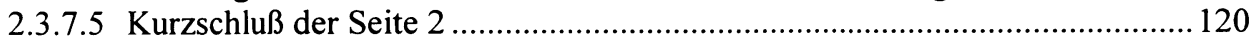

2.4 Nichtkosinusförmige periodische Wechselgrößen ………………………....... 121

2.4.1 Darstellung periodischer Wechselgrößen durch Fourierreihen ........................122

2.4.2 Symmetrien in der Kurvenform von periodischen Wechselgrößen ................... 124

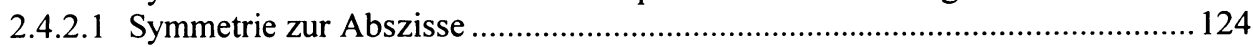

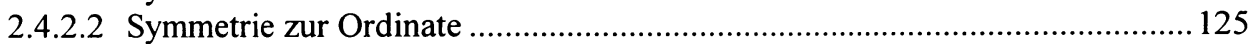

2.4.2.3 Verschiebung des Koordinatenursprungs.................................................... 126

2.4.3 Kenngrößen nichtkosinusförmiger periodischer Wechselgröße .........................126

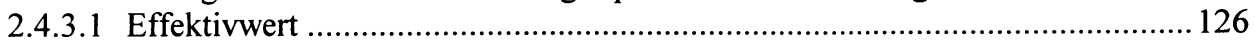

2.4.3.2 Grundschwingungsfaktor, Klirrfaktor, Formfaktor, Scheitelfaktor .................. 127

2.4.4 Anwendung an einer Zweipuls-Brückenschaltung ………………................. 128

2.4.4.1 Leerlauf der Brückenschaltung ……………………................................. 128

2.4.4.2 Belastung der Zweipuls-Brückenschaltung ................................................. 130

2.4.5 Netzberechnungen mit nichtkosinusförmigen Wechselgrößen.......................... 133

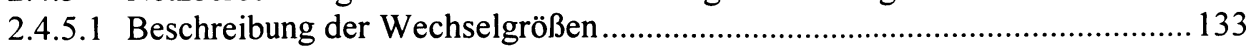

2.4.5.2 Nichtkosinusförmige Leerlaufspannung des Wechselstromnetzes .................... 134

2.4.5.3 Abnehmer als Konstantstromquelle für höhere Harmonische ……...................135

\section{TRANSFORMATIONEN FÜR DREIPHASENSYSTEME}

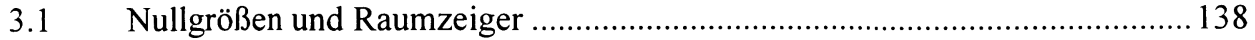

3.1.1 Definition der Nullgröße und des Raumzeigers ............................................ 138

3.1.2 Umrechnung von Raumzeigern zwischen beliebigen Bezugskoordinaten-

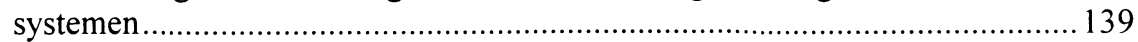

3.1.3 Rücktransformation von Raumzeigern und Nullgrößen ....................................141 
3.1.4 Raumzeiger, Diagonalkomponenten und Zwei-Achsen-Komponenten ........... 143

3.1.5 Momentane Drehstromleistung und normierte Transformationen.................... 146

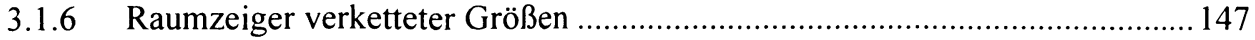

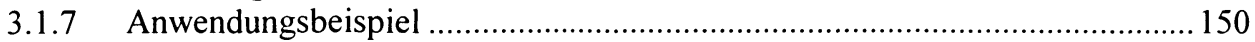

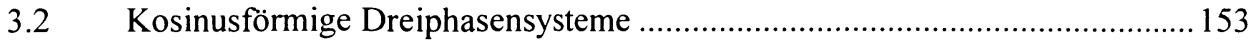

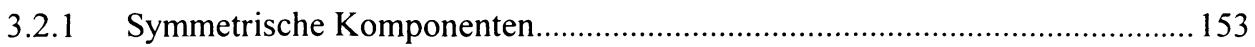

3.2.2 Raumzeiger und Symmetrische Komponenten.......................................... 155

3.2.3 Diagonal-Komponenten kosinusförmiger Dreiphasensysteme ..................... 159

3.2.4 Zwei-Achsen-Komponenten kosinusförmiger Dreiphasensysteme ................ 161

3.2.5 Verkettete kosinusförmige Dreiphasensysteme ......................................... 161

3.3 Nichtkosinusförmige periodische Dreiphasensysteme ................................. 163

3.3.1 Periodische Raumzeiger mit symmetrischen Zeitfunktionen.......................... 164

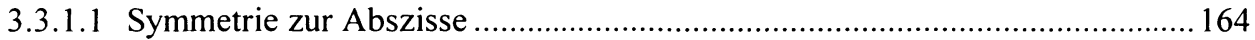

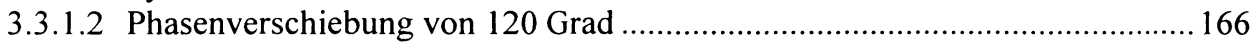

3.3.1.3 Symmetrie zur Abszisse und Phasenverschiebung von $120 \mathrm{Grad}$................... 168

3.3.2 Charakteristische Harmonische in Drehstromsystemen ................................ 169

3.3.2.1 Zweipulsbrücken als Abnehmer im Drehstromsystem ............................... 170

3.3.2.2 Magnetisierungströme von Drehstromtransformatoren ............................... 172

3.3.3 Ströme eines dreipulsigen Stromrichters in Mittelpunktschaltung .................. 176

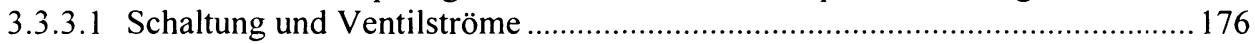

3.3.3.2 Raumzeiger der Ventilströme.............................................................. 178

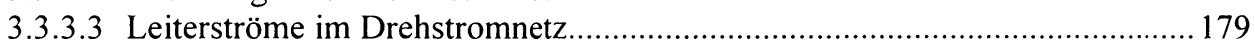

3.3.4 Überlagerung von p-pulsigen Raumzeigern .............................................. 180

3.3.4.1 Periodische Raumzeiger im synchron umlaufenden Koordinatensystem ........ 180

3.3.4.2 Überlagerung von zwei dreipulsigen Raumzeigern..................................... 182

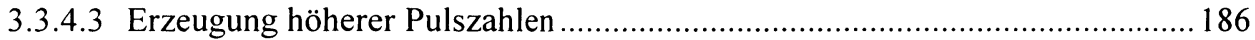

3.4 Transformation symmetrischer Drehstromnetze ............................................ 190

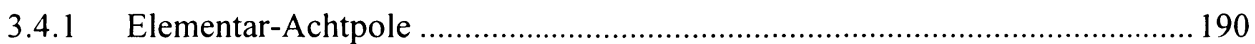

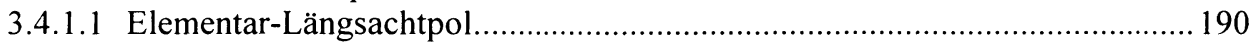

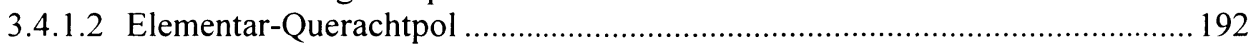

3.4.1.3 Elementar-Achtpole in Symmetrischen Komponenten................................... 193

3.4.2 Satz von der Ersatzspannungsquelle für Drehstromnetze ............................. 195

3.4.3 Ersatzschaltungen für Drehstromtransformatoren ......................................... 197

3.4.3.1 Drehstromtransformator mit unverschalteten Wicklungen .............................. 197

3.4.3.2 Verschaltung der Wicklungen eines Drehstromtransformators ......................200

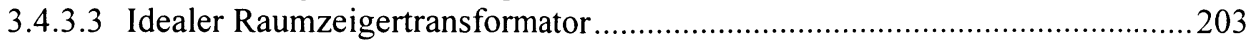

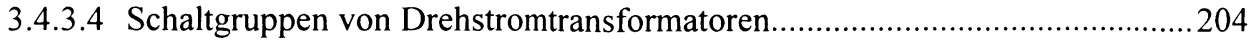

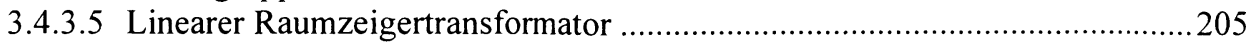

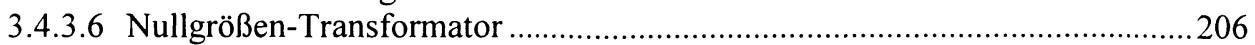

3.4.3.7 Drehstrom-Transformator in Symmetrischen Komponenten..........................210

3.4.4 Rotierende elektrische Maschinen.............................................................. 212

3.4.4.1 Besonderheiten im Vergleich zu anderen Betriebsmitteln ...........................2 212 
3.4.4.2 Induktivitäten und Flüsse von Dreiphasenwicklungen ................................213

3.4.4.3 Spannungsgleichungen der rotierenden elektrischen Maschine .....................217

3.4.4.4 Ersatzschaltungen rotierender elektrischer Maschinen im Mitsystem .............218

3.4.4.5 Ersatzschaltung rotierender elektrischer Maschinen im Gegensystem ............220

3.4.4.6 Ersatzschaltung rotierender elektrischer Maschinen im Nullsystem ...............222

3.4.4.7 Rücktransformation der Symmetrischen Impedanzen rotierender elektrischer Maschinen ......................................................................................... 223

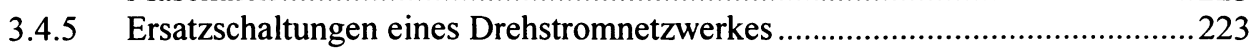

3.5 Unsymmetrische Betriebszustände in Drehstromnetzen................................227

3.5.1 Symmetrischer Betrieb eines Drehstromsystems......................................227

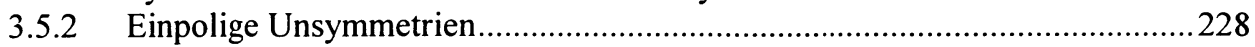

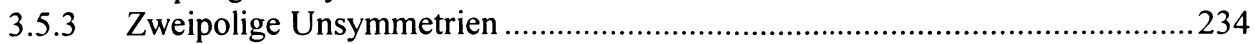

3.5.4 Unsymmetrische Last in Dreieck-Schaltung ............................................240

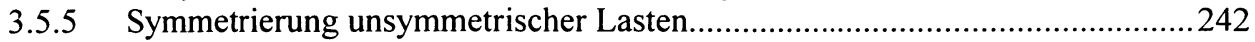

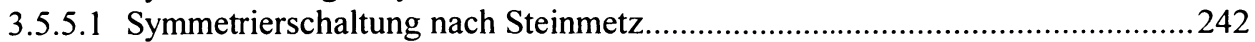

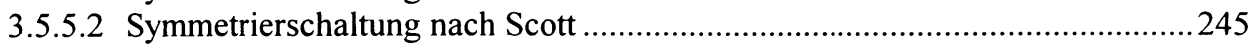

\section{LEISTUNGEN IN ELEKTROENERGIESYSTEMEN}

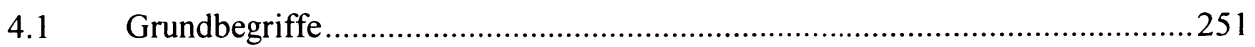

4.1.1 Wirkungsgrad energetischer Prozesse ....................................................2251

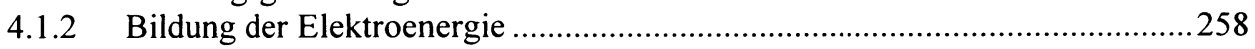

4.1.3 Verluste in elektrischen Energieversorgungsnetzen ..................................260

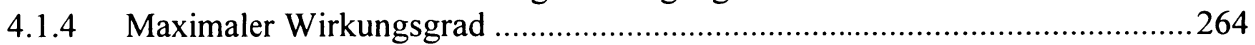

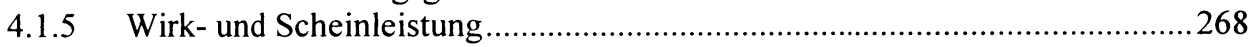

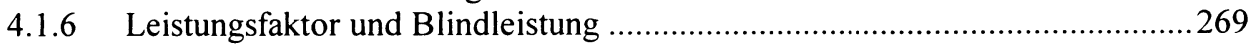

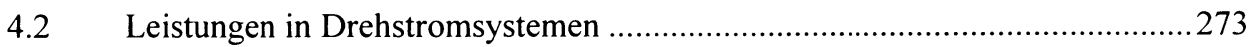

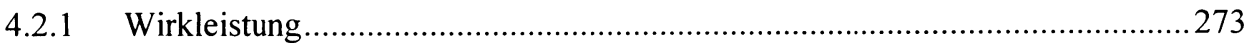

4.2.2 Effektivwerte von Dreiphasensystemen und Scheinleistung ........................2275

4.2.3 Leistungsverhältnisse bei kosinusförmig symmetrischen Spannungen............277

4.2.4 Leistungen bei kosinusförmigen symmetrischen Spannungen und Strömen ...278

4.2.5 Leistungsfluß über einen Netzzweig .....................................................281

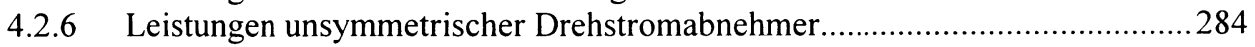

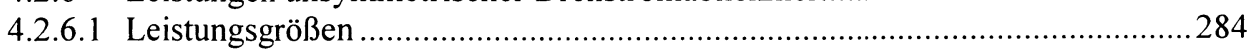

4.2.6.2 Berechnung der unsymmetrischen Spannungen und Ströme .........................286

4.2.6.3 Niederspannungstransformator mit unsymmetrischer Belastung...................28

4.2.6.4 Besonderheit unsymmetrischer Belastungen ...........................................293

4.2.7 Leistungen von Stromrichtern im Drehstromsystem ....................................297

4.2.7.1 Leistungen bei kosinusförmig symmetrischen Spannungen ..........................297

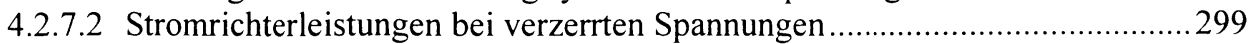

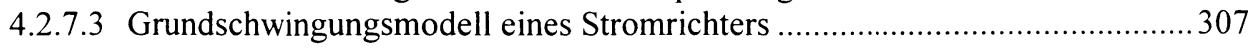




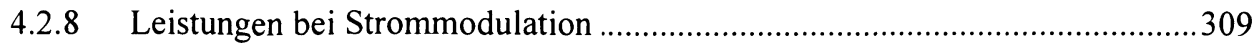

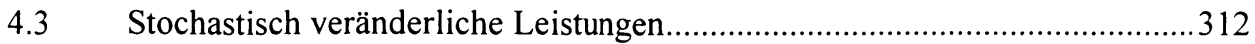

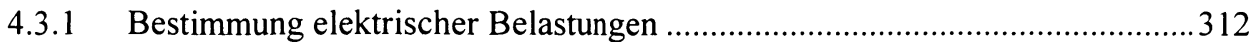

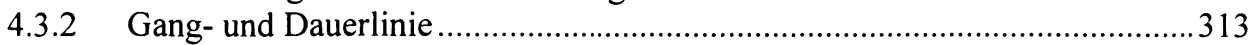

4.3.3 Weitere Kenngrößen zur Beschreibung von Belastungen ...............................319

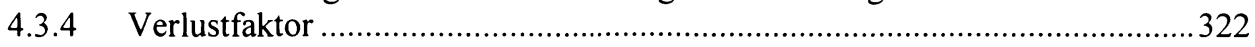

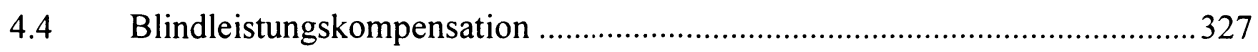

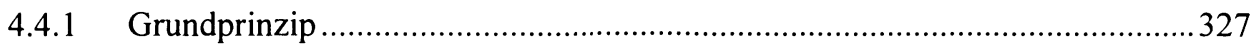

4.4.2 Kompensation der Verschiebungsblindleistung …………...........................329

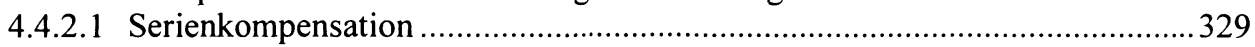

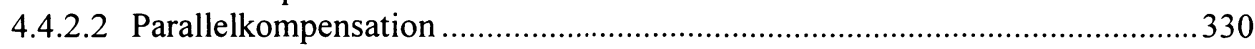

4.4.2.3 Parallelkompensation mit Synchronmaschinen ................................................331

4.4.2.4 Kompensation von Leitungen ................................................................333

4.4.3 Kompensation der Verzerrungsblindleistung ………….................................335

4.4.4 Kompensation der Modulationsblindleistung …………...............................335

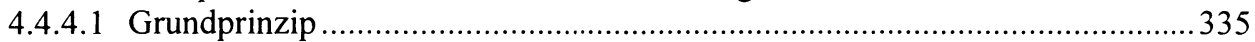

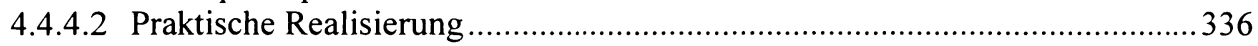

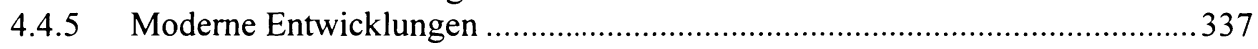

\section{WIRTSCHAFTLICHE ENERGIEVERSORGUNG}

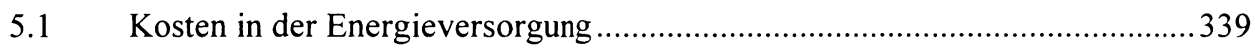

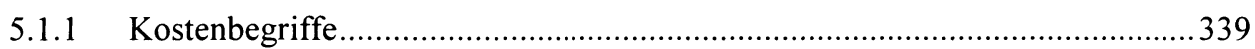

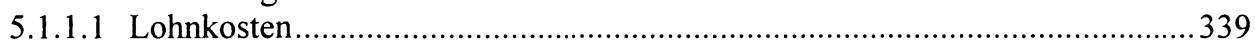

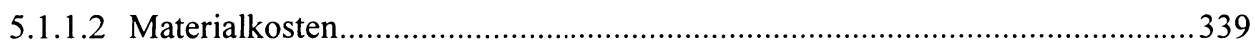

5.1.1.3 Anlagenkosten (Abschreibungen) ………………........................................339

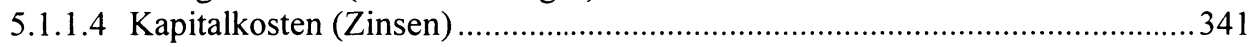

5.1.1.5 Sonstige Kosten (Gemeinkosten) .................................................................. 341

5.1.2 Feste und veränderliche Kosten ……………..............................................

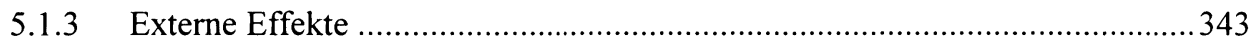

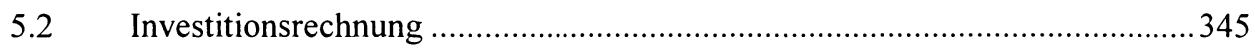

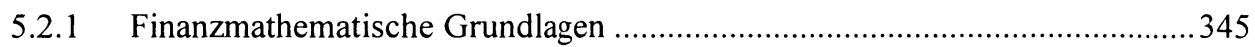

5.2.2 Gestehungskosten für die elektrische Energie .............................................348

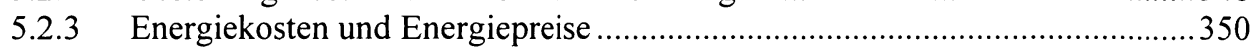

5.2.4 Bewertung von Verlusten in Energieversorgungsnetzen .................................353

5.3 Wirtschaftlicher Betrieb von elektrischen Energieversorgungsnetzen .............355

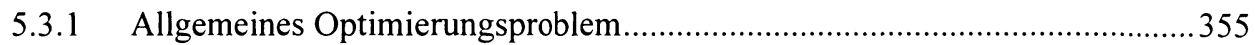

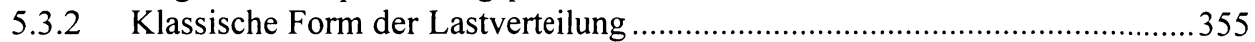

5.3.3 Verlustminimaler Verbundbetrieb bei konstanter Last.......................................360 
5.3.4 Ausgleich der Belastungskurve und wirtschaftlicher Kraftwerkseinsatz.........364

5.3.5 Wirtschaftliche Auslastung elektrischer Betriebsmittel................................368

5.3.5.1 Wirtschaftliche Auslastung von Transformatoren.........................................368

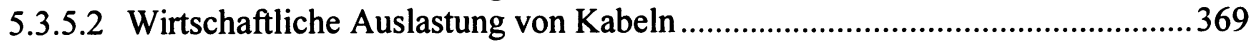

5.3.5.3 Wirtschaftliche Auswahl von Betriebsmitteln aus einer Typenreihe ................370

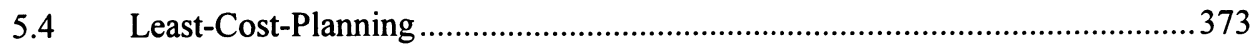

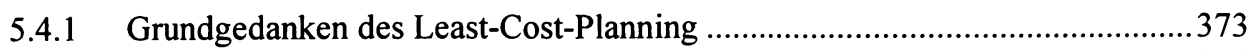

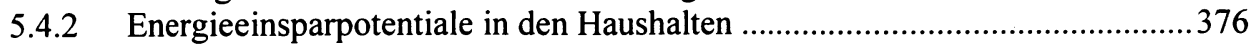

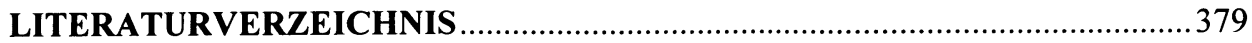

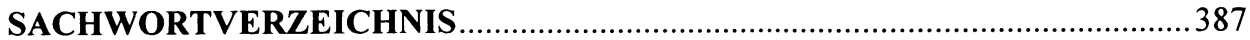

